\title{
Nuevo registro y ampliación de distribución geográfica para Agalychnis psilopygion (Anura: Hylidae: Phyllomedusinae) en el Chocó Biogeográfico de Colombia
}

\author{
New record and geographic distribution expansion for \\ Agalychnis psilopygion (Anura: Hylidae: Phyllomedusinae) in the \\ Chocó Biogeographic of Colombia
}

\author{
Pablo Palacios-Rodríguez ${ }^{1 *}$, Karen Quesada-Mosquera ${ }^{1}$, \\ Jhon Tailor Rengifo-Mosquera ${ }^{1}$
}

\begin{abstract}
Resumen
Se reporta una nueva localidad para Agalychnis psilopygion (Cannatella 1980), en la Parcela Permanente de Investigación Biológica (PPIB), ubicada en el centro del departamento del Chocó. Los tres individuos se encontraron asociados con un cuerpo de agua permanente producto de la intervención antrópica, en hojas de vegetación arbustiva, compartiendo hábitat con A. spurrelli, A. aff terranova, Hypsiboas pellucens, H. rubracylus, Craugastor fitzingeri, Dendrosophus phleobodes y Lithobates vaillantis. Este registro extiende rango la distribución geográfica para la especie en $190 \mathrm{~km}$ aproximadamente de la localidad tipo. El descubrimiento de esta especie listada por la Convención sobre el Comercio Internacional de Especies Amenazadas de Fauna y Flora Silvestre en el Apéndice II, además, carente de información ecológica, biológica y relaciones filogenéticas, en el área de influencia de la PPIB en el corregimiento de Salero, es de importancia en términos de conservación de los bosques para la preservación de los anfibios de las tierras bajas del Pacífico colombiano.
\end{abstract}

Palabras clave: Anuros, Colección biológica, Extensión de distribución, Hábitats.

\begin{abstract}
We report a new locality for Agalychnis psilopygion (Cannatella 1980) in Biological Research Permanent Plot ( PPIB ), located in the center of the department of Chocó; all three individuals were found associated with a permanent water body product human intervention in leaves of bushes, sharing habitat with $A$. spurrelli, A. aff terranova, Hypsiboas pellucens, H. rubracylus, Craugastor fitzingeri, Dendrosophus phleobodes and Lithobates vaillantis. This record extends the geographic range for the species about $190 \mathrm{~km}$ from the type locality. The discovery of this species listed by the Convention on International Trade in Endangered Species of Fauna and Flora in Appendix II also lacks ecological, biological and phylogenetic relationships in the area of influence of the PPIB in the village of Salero is important in terms of conservation of forests for the preservation of amphibians of the Colombian Pacific lowlands.
\end{abstract}

Keywords: Anura, Biological collection, Distribution of extension, Habitats.

Agalychnis psilopygion (Cannatella 1980), fue descrita basado en un macho adulto colectado en la vereda el Danubio, río Anchicayá. En Colombia la especie se distribuye en el flanco occidental de la cordillera Occidental, departamentos de Valle del Cauca y Cauca, entre los 100 y 500 metros de elevación (Cannatella 1980; Ruiz et al. 1996; Acosta 2000;
Lynch y Suárez 2004; Castro y Vargas 2008). La especie fue descrita inicialmente como Phyllomedusa psilopygion, luego fue clasificada dentro del género Hylomantis (sensu Faivovich et al. 2005) y posteriormente Faivovich et al. (2010) utilizando datos moleculares no apoyaron la monofilia del género Hylomantis, ubicando a sus miembros dentro del gé-

Grupo de Investigación en Herpetología, Facultad de Ciencias Naturales, Programa de Biología, Universidad Tecnológica del Chocó; Ciudadela Universitaria, Quibdó, Chocó, Colombia. e-mail: paparo92@gmail.com

Fecha recepción: Octubre 24, 2013 Fecha aprobación: Diciembre 7, 2013 Editor Asociado: Valois H. 
nero Agalychnis.

Agalychnis psilopygion (Figura 1) se caracteriza por presentar hocico corto, truncado en vista lateral y agudamente redondeado en vista dorsal; ojos grandes y protuberantes, pupila vertical y elíptica y membrana palpebral clara; brazo y antebrazo delgado, con pliegue ulnar débilmente desarrollado, extendiéndose del codo hasta el disco del cuarto dedo; dedos de longitud moderada y aplanados en sección, sin membranas interdigitales; presenta miembros posteriores largos y delgados. Se diferencia de la especie centroamericana A. lemur, por la ausencia de tubérculos paracloacales; además la superficie ventral de la ingle en A. psilopygion es débilmente granular, con tubérculos en A. lemur; además usualmente los individuos de $A$. psilopygion presentan verrugas dorsales blancas, nunca presentes en A. lemur (Cannatella 1980).

Aquí se presenta una extensión de distribución geográfica de A. psilopygion (Figura 2) basado en una hembra (COLZOOCH-H 1202; SVL $=46,2)$ y dos machos adultos (COLZOOCH-H 1203, SVL= 36,9; COLZOOCH-H 1204, SVL= 35,8). Los especímenes se depositaron en la Colección Científica de Referencia Zoológica de la Universidad Tecnológica del Chocó «Diego Luis Córdoba». Los individuos se colectaron en el área de influencia de la Parcela Permanente de Investigación Biológica (PPIB;

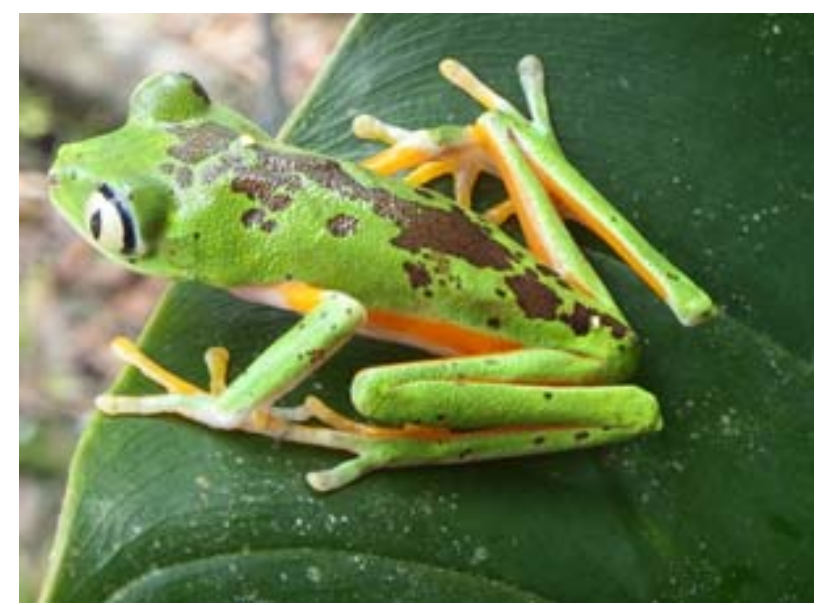

Figura 1. Agalychnis psilopygion en el área de influencia de la PPIB para el corregimiento de Salero, Municipio de Unión Panamericana, Chocó. Foto por Jhon Tailor Rengifo-Mosquera.
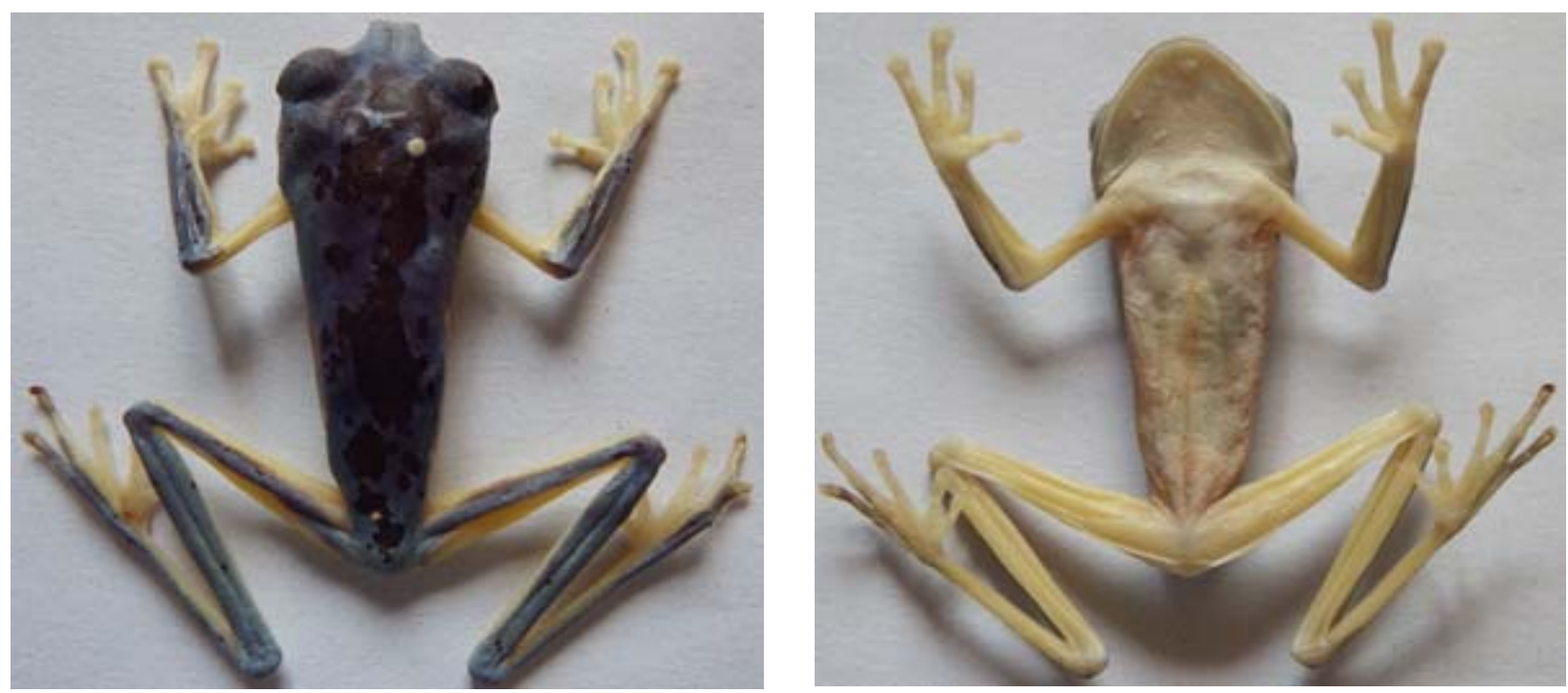

Figura 2. Vista dorsal (izquierda) y ventral (derecha) de un macho de Agalychnis psilopygion (COLZOOCH-H 1203) colectado en el corregimiento de Salero, departamento del Chocó, Colombia. Fotos por Pablo Palacios-Rodríguez 
$05^{\circ} 19^{\prime} 19,5^{\prime \prime} \mathrm{N}, 76^{\circ} 37^{\prime} 43,8^{\prime \prime} \mathrm{W}$, a 115 metros de elevación), en el corregimiento de Salero, municipio de Unión Panamericana, departamento del Chocó, Colombia (Figura 3). Esta zona de influencia es dominada por un bosque secundario, en donde históricamente se ha practicado extracción de oro y madera; estos individuos fueron encontrados aplicando el método de búsqueda por encuentros visuales (VES por sus siglas en inglés) (Crump y Scott 1994), durante una noche de lluvia, a las 19:45. Los individuos se encontraron en un parche de vegetación que rodeaba una pequeña charca, perchados al lado de sus posturas aproximadamente a tres metros de altura. Agalychnis psilopygion es simpátrica con A. spurrelli (Boulenger 1913), Agalychnis aff terranova; Hypsiboas rubracylus (Cochran y Goin 1970), $H$. pellucens (Werner 1901), Craugastor fitzingeri (Schmidt 1857), Dendrosophus phleobodes (Stejneger 1906) y Lithobates vaillantis (Brocchi 1908).
Nosotros reportamos aquí el primer registro de A. psilopygion para el departamento del Chocó, extendiendo su distribución en línea recta cerca de 190 km (Figura 3) de su localidad tipo y constituyendo el registro más norte hasta ahora para esta especie.

De acuerdo con Bolívar et al. (2004), A. psilopygion es una especie poco común, se conocen muy pocos datos sobre su extensión de presencia y hasta el momento se desconoce la tendencia de sus poblaciones. El descubrimiento de esta especie listada por la Convención sobre el Comercio Internacional de Especies Amenazadas de Fauna y Flora Silvestre en el Apéndice II, además, carente de información ecológica, biológica y relaciones filogenéticas, en el área de influencia de la PPIB en el corregimiento de Salero, es de importancia en términos de conservación de los bosques para la preservación de los anfibios de las tierras bajas del Pacífico colombiano.

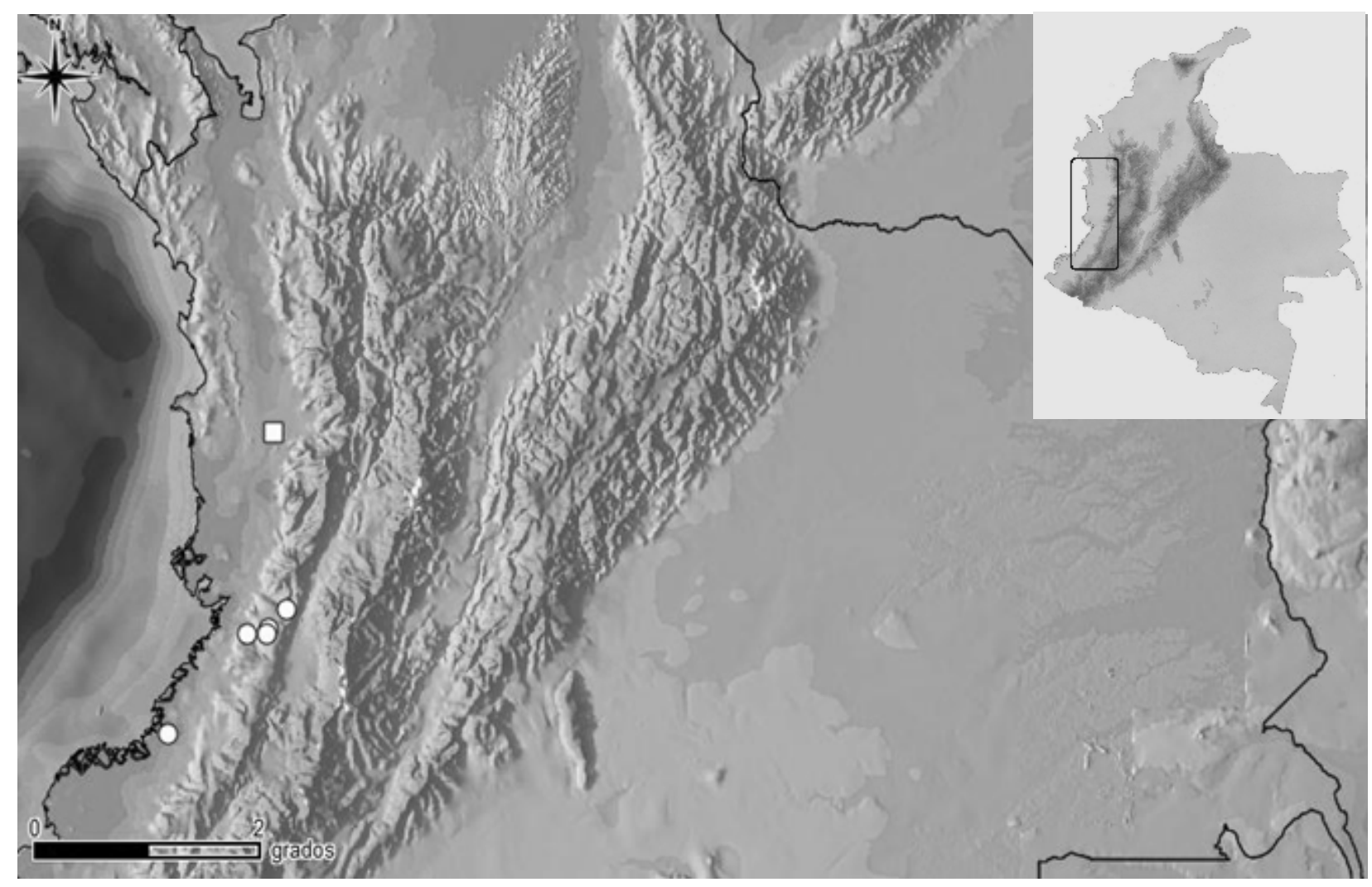

Figura 3. Distribución geográfica de Agalychnis psilopygion en el Chocó Biogeografico de Colombia. 


\section{Agradecimientos}

Los autores agradecen a la comunidad de Salero por su inmensa hospitalidad en todos estos años de visita y al grupo de Herpetología, Centro de Investigación en Biodiversidad y Hábitat. A la Vicerrectoría de Investigaciones de la Universidad Tecnológica del Chocó por la financiación y la grandiosa voluntad de investigación que siempre brindan. Al Doctor John D. Lynch y Mauricio Rivera por su orientación, confirmación de los ejemplares y comentarios en el manuscrito.

\section{Literatura citada}

Acosta AR. 2000. Ranas, salamandras y caecilias (Tetrapoda: Amphibia) de Colombia. Biota Colomb. 1 (3): 289-319.

Bolívar W, Jungfer KH, Rengifo JM. 2004. Hylomantis psilopygion. In: IUCN 2013. IUCN Red List of Threatened Species. Version 2013.1. Downloaded on 06 October 2013. URL: www.iucnredlist.org

Cannatella DC. 1980. A review of the Phyllomedusa buckleyi group (Anura: Hylidae). Ocassional Papers of the Museum of Natural History. The University of Kansas. 14611.
Castro F, Vargas F. 2008. Anfibios y reptiles en el departamento del Valle del Cauca, Colombia. Biota Colomb. 9 (2): 251-77.

Crump ML, Scott NJ. 1994. Visual Encounter Surveys. In: Measuring and monitoring biological diversity. Standard methods for amphibians. Eds. Heyer W, Donnelley MA, McDiarmid RA, Hayec LC, Foster MC. Washington DC: Smithsonian Institution Press.

Faivovich J, Haddad CFB, García PCA, Frost DR, Campbell JA, Wheeler WC. 2005. Systematic review of the frog family Hylidae, with special reference to Hylinae: phylogenetic analysis and taxonomic revision. Bull Am Mus Nat Hist. 294: 1-240.

Faivovich J, Haddad CFB, Baeta D, et al. 2010. The phylogenetic relationships of the charismatic poster frogs, Phyllomedusinae (Anura, Hylidae). Cladistics. 26: 227-61.

Lynch JD, Suárez A. 2004. Catálogo de anfibios en el Chocó Biogeográfico. p. 654-68. In: Rangel-Ch JO (ed.). Colombia Diversidad Biótica IV. El Chocó Biogeográfico/Costa Pacífica. Volumen I. Bogotá: Universidad Nacional de Colombia.

Ruiz PM, Ardila MC, Lynch JD. 1996. Lista actualizada de la fauna anfibia de Colombia. Revista de la Academia Colombiana de Ciencias Exactas, Físicas y Naturales. 20 (77): 365-415. 